\title{
Development and analysis of a model based on chirped fiber Bragg gratings employed for cracks characterization in materials
}

\author{
ANGÉLICA M. MESA YANDYa,b*, RICARDO DUCHOWICZ ${ }^{\mathrm{a}, \mathrm{b}}$, NÉLIDA A. \\ RUSSO $^{\mathrm{a}}$, JOSÉ L. CRUZ ${ }^{\mathrm{c}}$, AND MIGUEL V. ANDRÉS ${ }^{\mathrm{c}}$ \\ ${ }^{a}$ Centro de Investigaciones Ópticas (CONICET-CIC), Cno. Centenario y 506, (1897) Gonnet, Buenos Aires, Argentina \\ ${ }^{b}$ Facultad de Ingeniería, Universidad Nacional de La Plata, 1 y 47, (1900) La Plata, Buenos Aires, Argentina \\ ${ }^{c}$ Departamento de Física Aplicada - ICMUV, Universidad de Valencia, Burjassot 46100, Spain \\ *angelicam@ciop.unlp.edu.ar
}

\begin{abstract}
In this work a model was developed that allows to understand the behavior of a chirped fiber Bragg grating for the detection and characterization of cracks in materials. In addition to the amplitude response, we show that the group delay of the grating provides useful information for the characterization of the crack. The position of the crack can be determined thanks to the linear chirp of the grating that fixes a correlation between the spatial position and both, the wavelength and the group delay. However, our analysis shows that this simple approach has a source of error, which can be overcome if a controllable external strain can be applied to the embedded grating, additional to the strain generated by the embedding process. Thus, the width of the crack can be also estimated. The effect of the appearance of a crack on the grating generates simple o multiple transmission peaks that are analysed considering the behavior of a Fabry-Perot fiber cavity. This simple model was experimentally tested and preliminary results were in good agreement with the simulations.
\end{abstract}

Keywords: Optical fibre sensors, Modelling of sensors, Chirped fibre Bragg gratings, Fabry-Perot cavity, Identification of material cracks

\section{Introduction}

The interest of the aerospace industries in structural health and monitoring systems is continuously increasing. Among the techniques available in literature those based on Fiber Bragg Grating (FBG) sensors are much promising thanks to their peculiarities [1], [2] In comparison with the traditional mechanical and electrical sensors, the optical fiber sensors possess some unique advantages such as small size, light weight, immunity to electromagnetic interference (EMI) and corrosion, embedding capability, and therefore they have been employed in monitoring of engineering structures worldwide [3]. Bettini et al [1] started from a numerical model capable of simulating the spectral response of a grating subjected to a generic strain profile. While a standard uniform FBG can transduce only the average strain on its total length, a chirped one is able to provide direct information about the strain distribution profile along the grating itself. In fact, having a variable grating period, this kind of sensors has in principle a one-to-one correspondence between reflection spectrum wavelength and position on the perturbation [1]. Linear chirped Bragg gratings (linear CFBG) have a period that increases monotonously, the Bragg wavelength changes linearly with the position. Because of this, the reflectivity spectrum becomes broad. Under convenient conditions, the spectrum wavelengths can be related with the position along the grating. Thus, embedded CFBG have been proposed for the identification of crack location in composites [4], [5] and disbonding in composite joints [6]. More recently, an experimental study of fatigue damage development in composite patch repairs using CFBG has been reported [7]. These works based on CFBG extract the information from the measurement of the reflection spectra assuming the one-to-one correspondence between reflection spectrum wavelength and position on the perturbation.

(Reviewer1 - 1) In addition, some advances on chirped Bragg gratings in polymer fibers have been reported. Chirped fiber Bragg gratings were photoinscribed in undoped PMMA polymer optical fiber using an UV KrF excimer laser operating at $248 \mathrm{~nm}$. The evolution of the reflection spectrum was investigated as a function of the applied strain, temperature, and pressure, and one of the proposed 
applications was sensing transverse cracks in structural health monitoring [8], [9].

Nevertheless, an exhaustive study of the system has not yet been carried out. The contribution of the work presented here lies in the development of a rigorous model that allows a deep understanding of how a CFBG behaves when it is altered by the appearance of a crack in a material. This allows to determine not only the location of the crack but also its size. It is shown that, in addition to the conventional analysis of the amplitude response of the grating, it is possible to perform the aforementioned characterization using only the phase response i.e., the group delay spectrum of the grating. The CFBG is modeled by using the T-matrix method [10], [11]. In order to model the development of a crack, we assume that a differential strain will appear between the uniformly embedded part of the grating and the part of the grating that overlaps the crack. In addition, we show that if an external and controllable strain is applied to the embedded CFBG, then more detailed information can be obtained. After our rigorous analysis, we are able to provide a simple model in terms of an effective Fabry-Perot that accounts for most of the results obtained with the numerical simulations. This model takes into account the penetration of light in a reflecting FBG, which defines the effective length of a FBG [12]. Finally, some preliminary experimental results are also provided.

\section{Device Model}

A CFBG can be used to detect the appearance of cracks in materials (eg, polymers, compounds, cement, etc.), which can be part of structures such as bridges, buildings, roads, aerospace vehicles, automobiles, among others. These cracks can be generated during the manufacturing process or hardening (setting) of the material, by the action of loads applied to it, by aging of the structure or by accidental damage. To perform this detection, the grating can be embedded or superficially fixed on the material. The model proposed here allows the detection of cracks transverse to the grating, and in a first approximation it is considered that the fissure is generated in a certain instant and its size remains invariant. Specifically, this paper attempts to establish a technique for unambiguously determining the position and width of cracks that may appear in the material. The basic idea is to assume that, once the CFBG is embedded in a given material as for example a composite, it is subjected to a differential strain between the sections correctly embedded and the section that overlaps a crack. We assume that some strain $(\varepsilon)$ is generated in the material during its setting, and that cracks can appear in this process. Initially, the embedded CFBG will be subjected to an unknown strain, which will be different in the small portion of the grating overlapping the crack. Afterwards, we will consider also that an additional and calibrated strain can be applied to the CFBG, modulating the differential strain between the overall CFBG and the section overlapping the crack. Fig. 1 shows the gratings scheme considered in the analysis with a crack in $z=L / 2$, where $L$ is the total length of the CFBG.

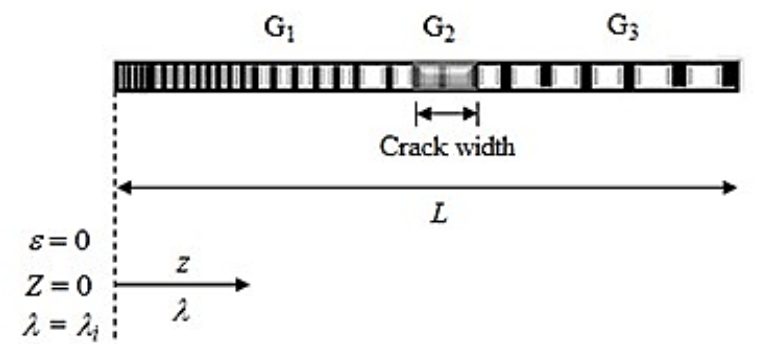

Fig. 1. Scheme of CFBG with a crack, as it is assumed in the simulations.

In order to simulate the differential strain in a simple way, we assume in our model that the grating portion overlapping the crack (G2) is strain free and its spectral properties remain unchanged, while the portions G1 and G3 will be affected by a uniform strain. Given the linear chirp of the CFBG, there is a bi-univocal relationship between the z-position and the corresponding wavelength variation, i.e. each spectral position of the grating corresponds to a spatial position $z$.

\section{Numerical Simulation}

For our numerical simulations, we consider a grating linearly chirped (Fig. 2). A defect (simulating a crack) is assumed in a given spatial position, for 
example in the middle of the grating, with different widths from 1 to $6 \mathrm{~mm}$. The original grating (without strain) has the following parameters: length $L=10$ $\mathrm{cm}$, refractive index amplitude modulation $\delta n=$ $2.3 \times 10^{-4}$, initial period $\Lambda_{i}=514 \mathrm{~nm}$, final period $\Lambda_{f}=$ $515.1 \mathrm{~nm}$, effective refraction index of the mode $n_{\text {eff }}$ equal to 1.5. The CFBG reflection band has the initial and final wavelengths $\lambda_{i}$ and $\lambda_{f}$ equal to $1542.31 \mathrm{~nm}$ and $1545.31 \mathrm{~nm}$, respectively, which determine a linear chirp $C=\left(\lambda_{f}-\lambda_{i}\right) / L=30 \mathrm{pm} / \mathrm{mm}$. Assuming a negligible fiber dispersion, the group velocity $v_{g}=\mathrm{c} /$ $n_{\text {eff }}$ determines the nominal group delay in reflection, per unit length $\left[\tau\left(\lambda_{f}\right)-\tau\left(\lambda_{i}\right)\right] / L=2\left(v_{g}\right)^{-1}=10 \mathrm{ps} / \mathrm{mm}$. Finally, the nominal chromatic dispersion of the grating when it is operated in reflection will be $\left[\tau\left(\lambda_{f}\right)\right.$ - $\left.\tau\left(\lambda_{i}\right)\right] /\left(\lambda_{f}-\lambda_{i}\right)=2\left(C v_{g}\right)^{-1}=333 \mathrm{ps} / \mathrm{nm}$.

Fig. 2 gives the computed reflectivity and group delay spectra for the CFBG, using the Transfer Matrix Method [10]. In it, we have included the average chromatic dispersion (white dashed line) that is obtained by fitting the central part of the plot. The slope of the average chromatic dispersion is 342 $\mathrm{ps} / \mathrm{nm}$ and the origin $\tau=0$ is at $\lambda=1542.39 \mathrm{~nm}$.

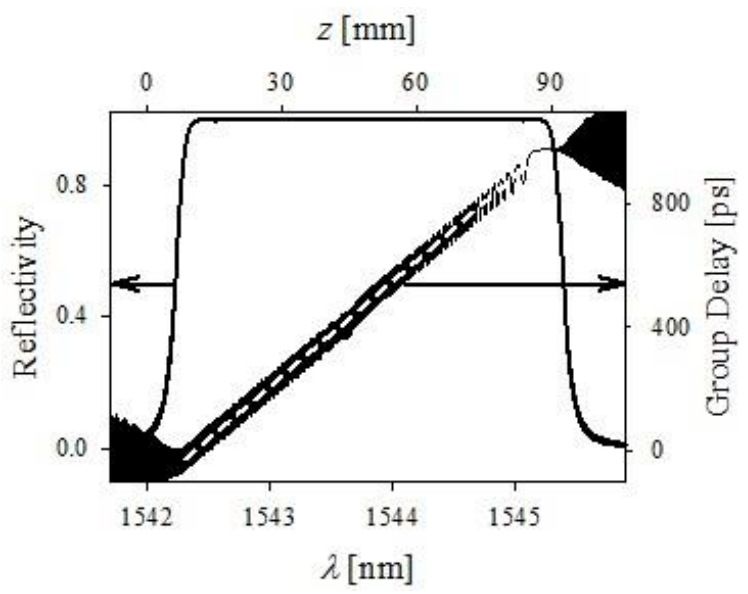

Fig. 2. Computed reflectivity and group delay for the initial CFBG. The white dashed line is the average chromatic dispersion that fits the numerical values.

As it will be shown later, when some strain is applied to the CFBG (over G1 and G3, because G2 is not affected) changes are observed on the reflection spectrum and the group delay of this system. We assume a standard dependence of the silica refractive index $\left(n_{\text {Silica }}\right)$ with the strain $(\varepsilon): \delta n_{\text {Silica }} / n_{\text {Silica }}=-p_{e} \varepsilon$, being $p_{e}=0.21[13]$.

\subsection{Spectral Characteristics}

Next, we will analyze the changes generated in the system due to the application of a strain. To understand how the deformation affects the behavior of the CFBG, in Fig. 3 the different spectral sections of the total grating are shown schematically versus wavelength. It is observed that, under a certain strain, two portions (G1 and G3, see Fig. 1) move in the same direction towards longer wavelengths. However, since the strain over G2 is zero, it does not shift with strain, but it overlaps with G1. While G1 and G3 are spectrally displaced by applying an external stress on the CFBG, G2 remains unchanged at its original spectral position. In addition, a spectral window appears between G1 and G3, from the absence of the contribution of G2 in the total spectrum of the grating (see Fig. 4). From a spectral point of view, a positive longitudinal deformation generated on gratings $\mathrm{G} 1$ and G3 (G2 is fixed), causes the reflectivity spectra of these two portions to be shifted to longer wavelengths as it is depicted in Fig. 3. The wavelength shift $\delta \lambda$ is proportional to the applied strain $(\varepsilon)$, whose origin can be either the material in which the CFBG is embedded or some external force. The relation between $\delta \lambda$ and $\varepsilon$ is given by [14]: $\delta \lambda / \lambda=\left(1-p_{e}\right) \varepsilon$. Thus, for our calculations we have assumed $p_{\varepsilon}=1-p_{e}=0.79$. Each wavelength of gratings $\mathrm{G} 1$ and G3 is displaced by considering $\lambda_{i \varepsilon}=$ $p_{\varepsilon} \varepsilon$.

The physical deformation of the grating is very small and can be neglected. The same happens from the spectral point of view, since the strain induces a similar shift for all grating portions, maintaining the same spectral width $\left(\Delta \lambda_{B} \approx \Delta \lambda_{B}{ }^{\prime}\right)$. Thus, apart from a wavelength shift, the slope of wavelength versus $\mathrm{z}$ position will remain constant, except for the spectral window opened by the crack. 


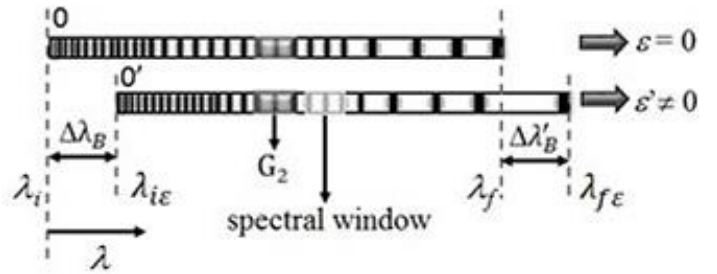

Fig. 3. Representation when the CFBG is strained.

Fig. 4 gives the computed reflectivity and group delay spectra corresponding to a defect size (G2) of $d$ $=1 \mathrm{~mm}$, located at $z=5 \mathrm{~cm}$ from the left edge of the grating $(z=0)$, and a strain $\varepsilon=291 \mu \varepsilon$. The differential strain between the crack and the rest of the fiber generates a resonance that gives rise to a narrow transmission window and a group delay peak whose spectral location is correlated with the spatial position of the simulated crack.

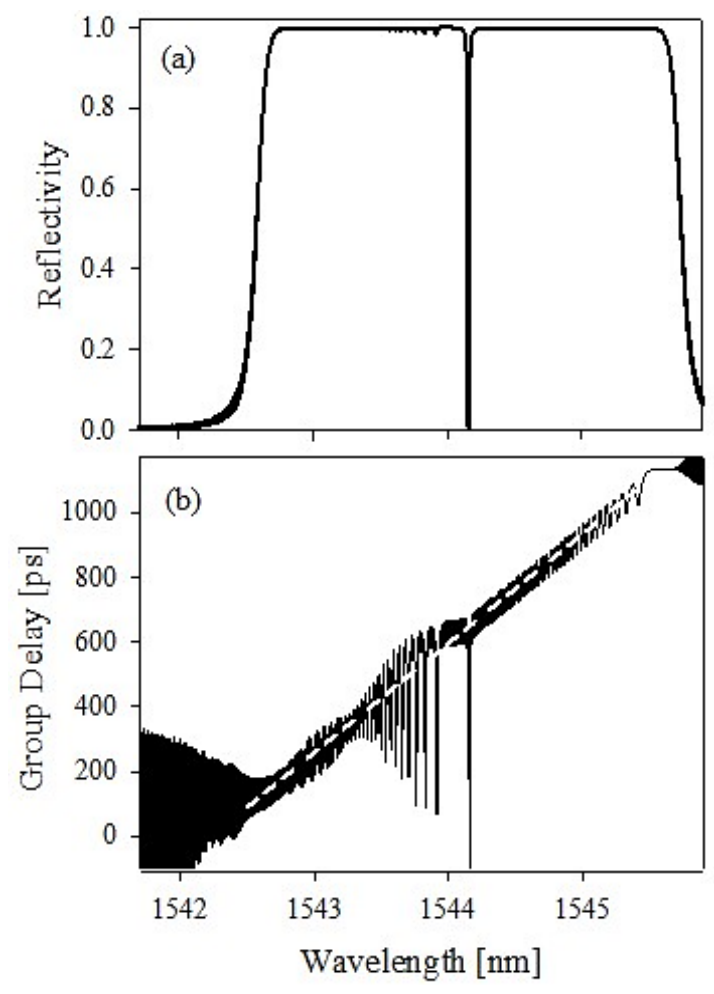

Fig. 4. CFBG with a defect of $1 \mathrm{~mm}$ : (a) reflection spectrum and (b) group delay. The white dashed line is the average chromatic dispersion that fits the numerical values.
Since the spectrum of the unperturbed grating G2 is now superimposed with grating G1, some interference peaks can be observed on the left of the dominant resonance, i.e. the shorter wavelengths. In Fig. 4 (b), the average chromatic dispersion obtained with the fitting to the numerical results is $342 \mathrm{ps} / \mathrm{nm}$.

In Fig. 5 we compare the reflectivity and group delay peaks for the case $d=1 \mathrm{~mm}, \varepsilon=291 \mu \varepsilon$. We can observe that are centered at the same wavelength, being narrower the group delay peak. The spectral width of the reflectivity peak for a crack of $1 \mathrm{~mm}$ is $7.0 \mathrm{pm}$ while the corresponding group delay peak width is $1.0 \mathrm{pm}$. Thus, the spectral position of the two peaks coincide, but the peak of the group delay will provide, in principle, better resolution in determining the crack position by a factor 5 to 7 . However, we will discuss this issue with more detail later in this work.

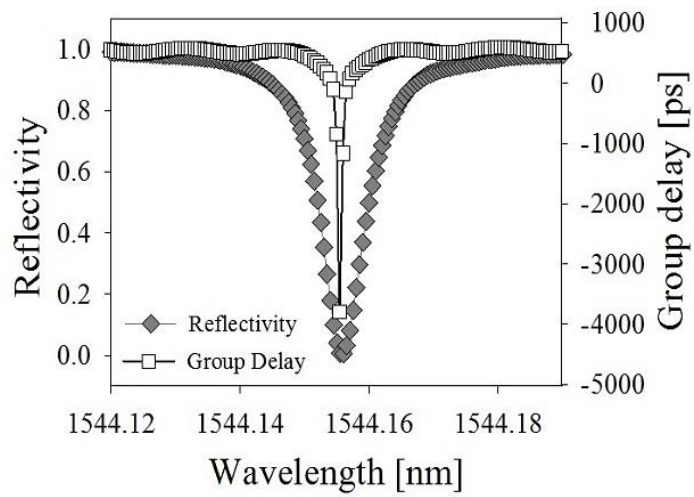

Fig. 5. Reflectivity and group delay peaks for $d=1$ $\mathrm{mm}$ and $\varepsilon=291 \mu \varepsilon$.

\subsection{Crack Position}

Our numerical results show that by measuring the spectral position of the resonant peaks with respect to the edge of the chirped grating, we can locate the position of a crack independently of the existing strain. Fig. 6 shows the reflection and group delay spectra for defects located at $L / 3$ and $2 L / 3$, both for the same strain value $(291 \mu \varepsilon)$. We find a good correlation between the wavelength of the notch that appears in the spectrum and the spatial location of the 
defect, i.e., the $z$ coordinates of the crack. This is the basic idea of using a CFBG. In order to confirm this simple relationship between the spectral response and the spatial location of the crack, independent of the strain, we define $\Delta \lambda=\left(\lambda_{p}-\lambda_{i \varepsilon}\right)$, where $\lambda_{p}$ and $\lambda_{i \varepsilon}$ correspond to the wavelengths of the transmission peak and the left edge of G1 grating, respectively.
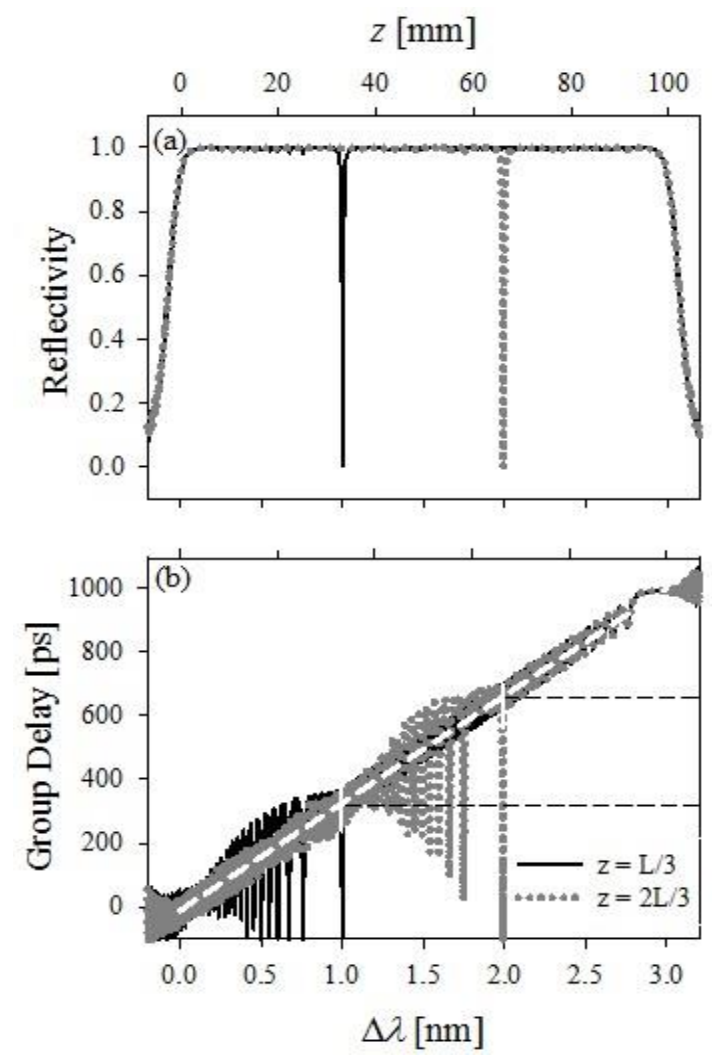

Fig. 6. Spectra for $1 \mathrm{~mm}$ cracks in different positions: $L / 3$ and 2L/3: (a) Reflectivity and (b) group delay, both computed for $\varepsilon=291 \mu \mathrm{s}$. The white line is the average chromatic dispersion.

When a strain is applied, the complete grating shifts to longer wavelengths and both values $\left(\lambda_{p}\right.$ and $\lambda_{i \varepsilon}$ ) change, but the difference is determined by the spatial position of the crack. It is worthwhile to mention that we could take the reference on the right edge on grating G3 $\left(\lambda_{d \varepsilon}\right)$ and define $\Delta \lambda^{\prime}$ as $\lambda_{d \varepsilon}-\lambda_{p}$. This option could have the advantage of avoiding the interference between G2 and the left border of G1 for high values of the strain.
For the analysis of the group delay information (see Fig. 6 (b)), we found useful to define what we have called the group delay position of the resonance $\left(\tau_{g}\right)$. This parameter is the group delay value that the original CFBG with no strain would have at the wavelength where the resonance appears due to the presence of the crack.

In order to make more clear the definition, we include in Fig. 6 (b) the white dashed line that corresponds to the average group delay, and with dashed horizontal lines we point to the intersections of the resonances produced by the crack. The intersections define the $\tau_{g}$ values of these two cases: 319 ps and 658 ps for crack positions at $z=L / 3$ and $z=2 L / 3$, respectively.

Fig. 7 shows the wavelength position $\left(\Delta \lambda_{p}\right)$ and the group delay position $\left(\tau_{g}\right)$ of the resonance, computed for different $\mathrm{z}$ position of the crack. We include in this plots the values computed for two strains $(291 \mu \varepsilon$ and $691 \mu \varepsilon$ ), in order to show that the results are in principle independent of strain, although later we will show that this is not exactly true. The relationship between the spatial coordinate $z$ and these two parameters $\Delta \lambda_{p}$ and $\tau_{g}$ is approximately linear and falls on the straight line defined by the nominal chirp, for both strain values. In Fig. 7 (a) the slope of the straight line is $1 / C$ (with $C=30 \mathrm{pm} / \mathrm{mm}$, the nominal value), and in Fig. 7 (b) the slope of the plotted line is $v_{g}=2 \times 10^{8} \mathrm{~m} / \mathrm{s}$. Thus, as a preliminary conclusion, we could say that in an experiment where the position of the crack should have to be determined, the measured values obtained for $\Delta \lambda_{p}$ and $\tau_{g}$ would determine the position of the crack regardless of the strain, since we can observe in the simulations that the points overlap the straight line. In principle, since the group delay peak is narrower, this would provide better resolution.

However, one can observe some deviations of the points obtained with numerical simulations from the straight lines that correspond to the nominal values of $C$ and $v_{g}$. These fluctuations are not numerical noise, but variations with an important physical meaning that one can find out when performing detailed simulations as a function of strain. We will discuss this in the next section. Moreover, for relatively wide cracks i. e., large values of $d$ the transmission spectra present several peaks, although there is a single crack in the CFBG (see Fig. 8). One can expect that this 
fact will increase the uncertainty in the spatial location of the crack from the spectral information. These bunches of peaks are present in previously reported experiments (see for example Fig. 13 in [4] and Fig. 4 in [7]). In some cases, they are reported as somewhat surprising double peaks associated with a single crack, and sometimes they are directly interpreted as corresponding to several cracks. Again, these bunches of peaks generated by a singlewide crack will behave in a particular and characteristic way when applying an external controllable strain.
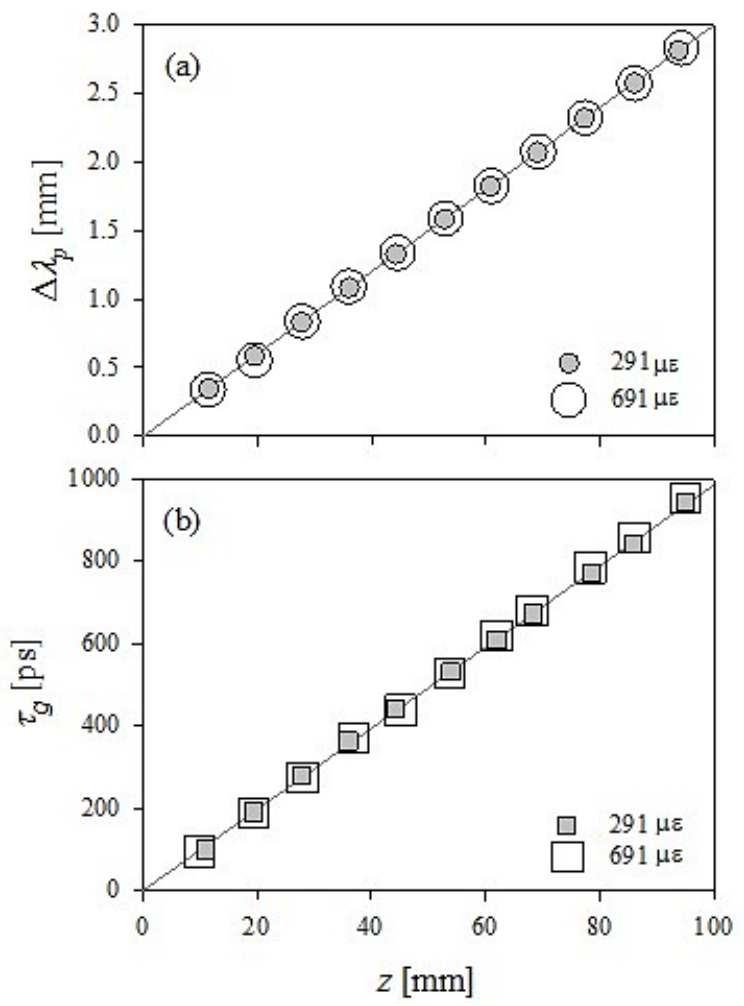

Fig. 7. Position of the crack (z) versus (a) wavelength position $\left(\Delta \lambda_{p}\right)$ and (b) group delay position $\left(\tau_{g}\right)$ of the resonance. We include the points computed for two strains values, $291 \mu \varepsilon$ and $691 \mu \varepsilon$.

\section{Response versus strain}

\subsection{Determination of crack width}

Numerical simulations of reflectivity and group delay spectra as a function of strain show up a relatively complex behavior of the device.

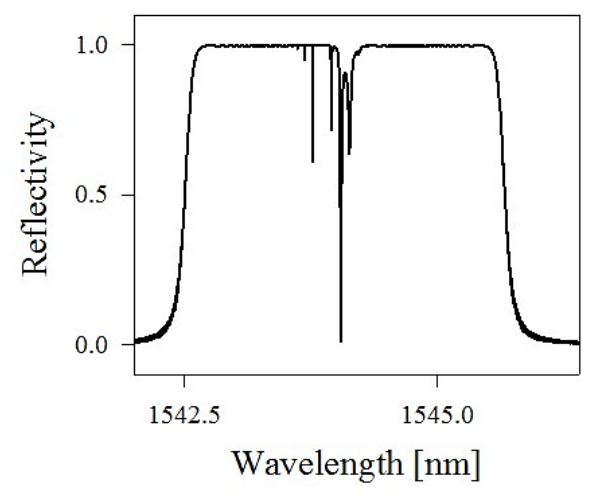

Fig. 8. Transmission spectra computed for CFBG with a crack located at $L / 2$ and a relatively large width of 6 mm.

Fig. 9 shows the wavelength of the resonant peak generated by the crack and its amplitude versus strain, for $d=1 \mathrm{~mm}$. If we analyze first the wavelength dependence with the strain (Fig. 9a), we find that the response has a staircase shape with an average slope that matches perfectly the shift of a FBG with strain, in good correspondence with the value that was considered for $p_{\varepsilon}$ : 0.79. If we now analyze the evolution of the peak amplitude versus strain (Fig. 9b) we find that the response is periodical and the period $T_{\varepsilon}(433 \mu \varepsilon$ for $d=1 \mathrm{~mm})$ matches the height of the steps observed in Fig. 9a. Before analyzing these results in more detail, we found necessary to explain the peculiar response of the device that we have just reported, in order to understand the physical origin of the staircase shaped response. In order to introduce a physical description of the crack effects, we will consider in a first approximation that the undisturbed section of the CFBG defines a Fabry-Perot with the anterior and posterior gratings (strictly speaking, it would be with the spectral end sides of gratings G1 and G3, since their Bragg wavelengths do not match).

The actual spectral response of the grating fragment G2 that forms the defect in the original CFBG participates in almost nothing and its spectral response ends up coming out of the spectrum of interest for high strain values. Therefore, what one could expect is that gratings G1 and G3 would shift linearly as a function of strain and that, in principle the peak associated with the crack would follow the 
shift of G1 and G3. In fact, this is what is observed in a large range of strain if we just consider the average slope, or if we focus our attention only on the peaks with maximum amplitude. The inset in Fig. 9a shows the slopes that are obtained for different values of $d$ in the lower range of strain values.
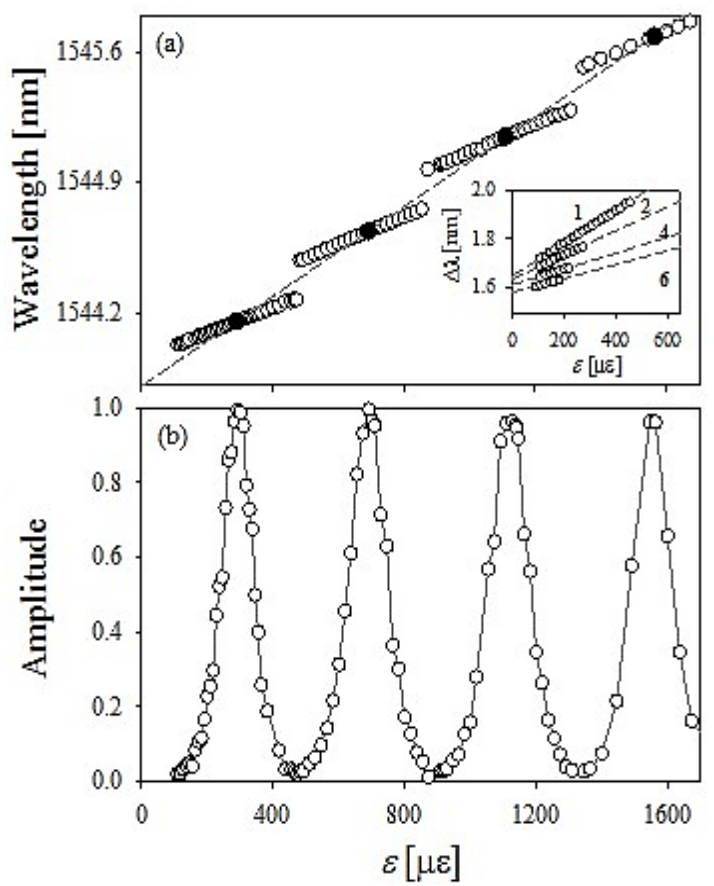

Fig. 9. (a) Strain versus wavelength of the transmission peak; the deepest peak of each cycle is in black. (b) Amplitude of the transmission peak versus strain. Both plots correspond to the case $d=1 \mathrm{~mm}$. The inset depicts the slopes of the first cycle, labeled with the width values $(d=1,2,4$ and $6 \mathrm{~mm})$.

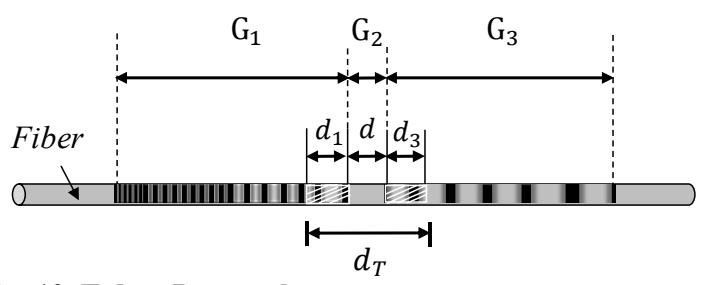

Fig. 10. Fabry-Perot scheme.

In Fig. 10, we give a scheme of the Fabry-Perot defined by the unperturbed section of CFBG (G2), where the crack is located and gratings G1 and G3. These two gratings are characterized by their effective lengths [12] $d_{1}$ and $d_{3}$ associated to the light penetration in both gratings $\left(d_{1}+d_{3}=d_{0}\right)$. The total effective length of the Fabry-Perot cavity is $d_{T}=d+$ $d_{0}$. It should be noted that a wavelength independent effective length might be considered only for CFBG segments with negligible chirp, i.e., relatively short segments around the section of the CFBG overlapping the crack, as it is in our case.

Changes due to strain on the CFBG generate a local differential decrease of the index $(\delta n<0)$ with respect to the effective index of gratings G1 and G3, since the effective index increases with strain according to the $p_{\varepsilon}$ coefficient $\left(p_{\varepsilon}>0\right)$. In other words, the defect generated by the crack is deeper as the strain is increased, in terms of effective refractive index. Thus, the phase difference generated by the defect will be proportional to $\delta n$ and $d$. Since the refraction index change is proportional to the applied strain, we will have:

$$
\delta \phi \propto-d \delta \varepsilon
$$

This $\delta \phi<0$ causes any Fabry-Perot resonance to shift to short wavelengths as $\delta \phi$ increases in absolute value. The spectral window in which the local FabryPerot operates with good visibility is limited by the spectral overlap of gratings G1 and G3. Normally, only a single resonance appears in the reflectivity spectrum. The free spectral range (FSR) of the FabryPerot $\left(\Delta \lambda_{0}\right)$ corresponds to each $2 \pi$ cycle of $\delta \phi$. As $\delta \phi$ decreases, every resonance shifts towards shorter wavelengths and, when it reaches the limit of the FSR, then a new resonance appears on the longer wavelengths side [15]. This produces each one of the steps observed in the simulations. Eventually, if $d$ increases and the FSR is sufficiently small, then we can observe two or three resonances simultaneously in the reflection spectrum of the CFBG (as Fig. 8 reports). If we define the phase $\varphi$ as the angle $\phi$ modulo $2 \pi$ (i.e. the phase $\varphi$ is the remainder when dividing $\phi$ by $2 \pi$ ), we can write the spectral shift $\left(\delta \lambda^{\prime}\right)$ due to the change of phase $\delta \phi$ as:

$$
\delta \lambda^{\prime}=-\Delta \lambda_{0} \frac{\delta \varphi}{2 \pi}, \quad \varphi \in[0,2 \pi]
$$

The value of $\Delta \lambda_{0}$, the Fabry-Perot Free Spectral Range, depends inversely to the effective length $(d+$ $\left.d_{0}\right)[12]$ : 


$$
\Delta \lambda_{0} \propto \frac{1}{\left(d+d_{0}\right)}
$$

Combining equations (1-3), we can write the shift of the resonance $\delta \lambda^{\prime}$ generated by the change of phase $\delta \varphi$ in the form:

$$
\delta \lambda^{\prime}=-B \frac{\lambda_{p} d \delta \varepsilon}{\left(d+d_{0}\right)}
$$

where $B$ is a constant that combines the factors of proportionality defined in eq. (1) and (3) divided by $2 \pi$ and $\lambda_{p}$. In order to account for the overall shift $\delta \lambda$, we have to add the shift produced by the strain in the CFBG. Thus, we obtain the following expression for the normalized slope $p_{d}$ :

$$
p_{d}=\frac{\delta \lambda}{\lambda_{p} \delta \varepsilon}=p_{\varepsilon}-B \frac{d}{\left(d+d_{0}\right)}
$$

In order to test this result, we computed the slope $p_{d}$ for different widths $d$, for the first step, i.e., small values of strain, as reported in the inset of Fig. 9 (a) (see Table 1). The trace corresponding to $d=5 \mathrm{~mm}$ was not drawn in the mentioned inset, for clarity of the figure.

\begin{tabular}{|c|c|}
\hline $\boldsymbol{d}[\mathbf{m m}]$ & $\boldsymbol{p}_{\boldsymbol{d}}[\mathbf{1} / \boldsymbol{\varepsilon}]$ \\
\hline $\mathbf{1 . 0}$ & 0.4485 \\
\hline $\mathbf{2 . 0}$ & 0.3144 \\
\hline $\mathbf{4 . 0}$ & 0.2075 \\
\hline $\mathbf{5 . 0}$ & 0.1838 \\
\hline $\mathbf{6 . 0}$ & 0.1703 \\
\hline
\end{tabular}

Table 1. Computed $p_{d}$ values for different crack sizes.

In Fig. 11 we plot $\left(p_{\varepsilon}-p_{d}\right) / d$ versus $1 /\left(d+d_{0}\right)\left(p_{\varepsilon}=\right.$ 0.79 ) and we find a perfect linear dependence by taking $d_{0}=1.2 \mathrm{~mm}$, with $B=0.753$ and zero yintercept, demonstrating that the Fabry-Perot model developed in this section appears to be physically correct. Equation (5) explains the slope difference between the average value $p_{\varepsilon}$ and the actual value $p_{d}$ obtained with the simulations. In addition, the model explains the staircase shape in terms of the phase modulo $2 \pi$ of the defect generated by the crack.

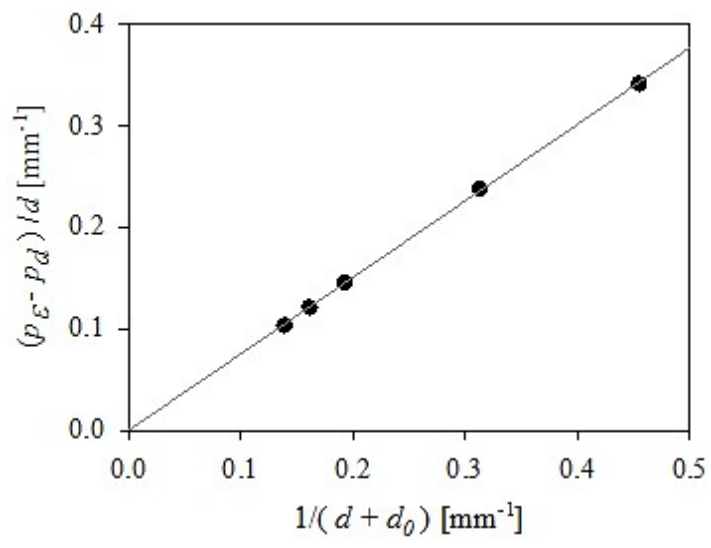

Fig. 11. Dependence of the slope deviation with respect to $p_{\varepsilon}$ normalized with $d$, on $\left(d+d_{0}\right)^{-1}$.

Once we have the numerical simulations and a physical model, we can propose two approaches to determine the width of a crack. We will assume that some initial strain exists, produced in the embedding process of the CFBG, and, furthermore, we will assume that we are able to apply an additional strain $\delta \varepsilon$ to the device in a controllable way. Thus, if we can measure the actual value of the slope $p_{d}$, then $d$ can be determined using the calibration of Fig. 11 that corresponds to eq. (5).

Alternatively, if the strain period that defines the steps, $T_{\varepsilon}$, can be measured, then the width $d$ of the crack can be determined using the calibration provided by the numerical simulations and summarized in Table 2 and Fig. 12. We see that it is a good approximation to consider a linear dependence of $T_{\varepsilon}$ with $\left(d+d_{0}\right)^{-1}: T_{\varepsilon} \approx C /\left(d+d_{0}\right), C=786 \mu \varepsilon / \mathrm{mm}$. Finally, using $d$ as a free parameter, one could numerically find the best fitting within a given strain range provided by the experimental data, by adjusting the response depicted in Fig. 9 (a).

\begin{tabular}{|c|c|c|}
\hline $\boldsymbol{T}_{\boldsymbol{\varepsilon}}[\boldsymbol{\mu} \boldsymbol{\epsilon}]$ & $\boldsymbol{d}[\mathbf{m m}]$ & $\mathbf{1} / \boldsymbol{d}+\boldsymbol{d}_{\mathbf{0}}\left[\mathbf{m m}^{-\mathbf{1}}\right]$ \\
\hline $\mathbf{3 5 5}$ & 1 & 0.4545 \\
\hline $\mathbf{2 5 5}$ & 2 & 0.3125 \\
\hline $\mathbf{1 5 5}$ & 4 & 0.1923 \\
\hline $\mathbf{1 0 9}$ & 5 & 0.1613 \\
\hline $\mathbf{1 0 0}$ & 6 & 0.1389 \\
\hline
\end{tabular}

Table 2. Computed $T \varepsilon$ values for different $d$ values. 


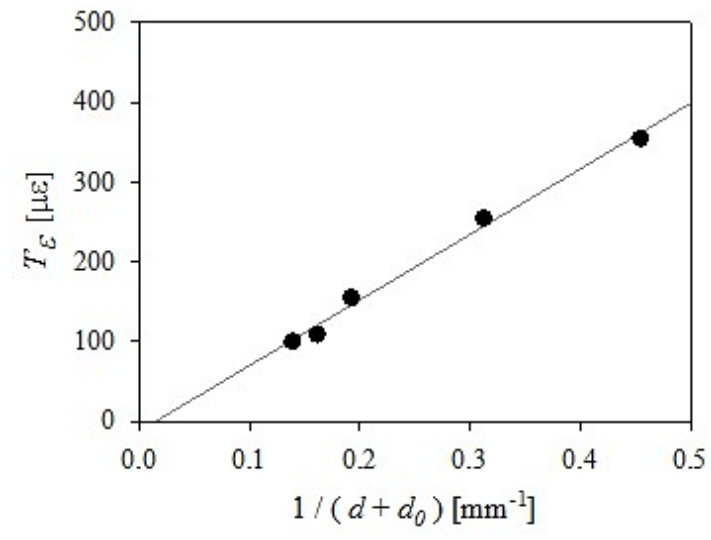

Fig. 12. Dependence of the strain period with respect to effective cavity width.

Similarly, to the amplitude response of the CFBG, we can analyze the group delay response.

We should stress here that the value of the group delay in the resonance condition obtained directly from the simulations is called $\tau_{g p}$, while $\tau_{g}$ is the group delay at the spectral position of the resonance peak, but measured on the curve corresponding to the slope of the chirp (see the definition in Fig. 6). Thus, Fig. 13 shows the behavior of these parameters versus strain, for a crack of $d=1 \mathrm{~mm}$ located at the center of the CFBG.

If we analyze $\tau_{g}$ versus strain, we see that the response is saw-tooth shaped with an average value that is constant. The expected value, according to the basic relationship depicted in Fig. 7 (b), would be 500 ps. We see that the actual value oscillates around that value due to the Fabry-Perot effect. This effect has the same physical origin as the staircase shape described for the amplitude response of the CFBG. Regarding Fig. 13 (b), we find a periodic behavior for $\tau_{g p}$ versus strain. The period is $T_{\varepsilon}$, the same that we observed in the amplitude response. Around the strain value in which the amplitude response is higher, we observe the characteristic group delay oscillation of a resonance. This strong oscillation helps to perform an accurate experimental determination of $T_{\varepsilon}$ and $d$.
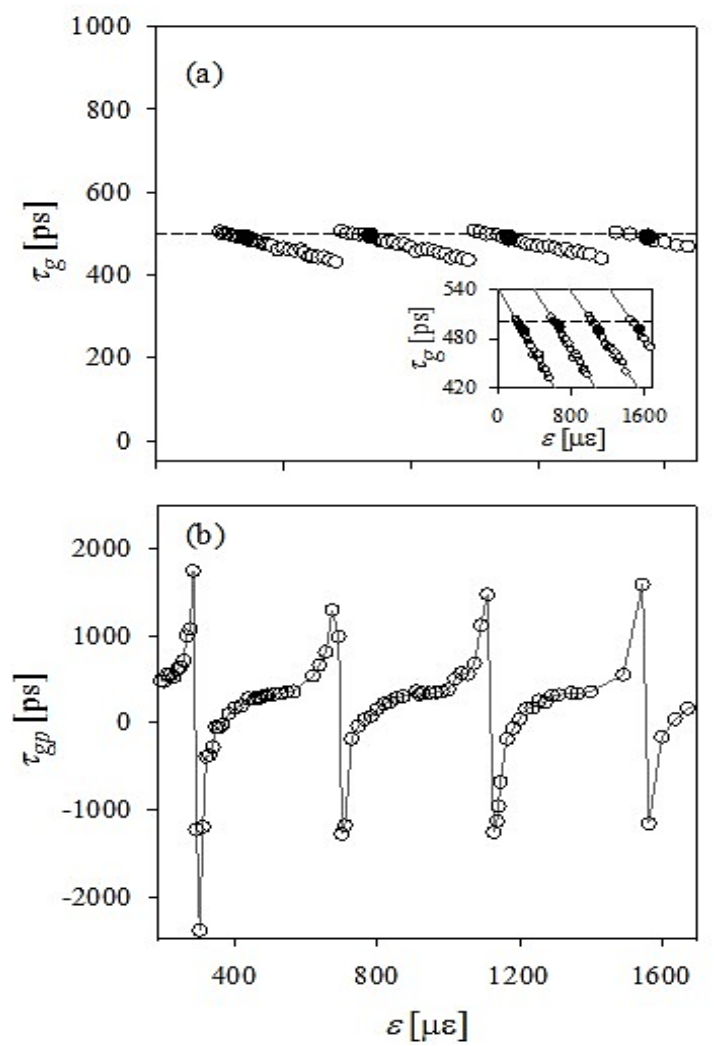

Fig. 13. (a) Dependence of group delay position $\left(\tau_{g}\right)$ versus applied strain. The deepest peak of each cycle is in black; the dashed line shows the group delay position that one could expect from Fig. 7 (b); the inset shows an enlargement of the plot. (b) Group delay value at the resonance $\left(\tau_{g p}\right)$ versus applied strain. Both plots correspond to the case $d=1 \mathrm{~mm}$.

\subsection{Fine determination of crack position}

After the analysis carried out in section 4.1, there are two important consequences for the determination of crack position using CFBG. First one is the fact that the amplitude of both reflectance and group delay peaks might be small, depending on the actual value of strain, and perhaps could not be detected experimentally. Therefore, in some reported experiments [4]-[7] they might be missing some cracks because of the specific strain that the CFBG might be supporting when recording the spectrum. In addition, after Fig. 8, we know that for broad cracks we may observe two or three peaks in the reflection spectrum that are produced by a single crack, as it 
can be observe clearly in some experiments previously reported in [4]-[7], for example.

At this point, we would like to stress the importance of being able to carry out a characterization versus applied strain. We can discuss here how the staircase response depicted in Fig. 9 (a) limits the precision when locating the position of a crack. In section 3.2 we assumed that the spectral position of the resonance was determined directly by the chirp of the grating, but after the rigorous simulations we know that the response is more complex. If we analyse Fig. 9 (a) we conclude that the maximum deviation of $\lambda_{p}$ with respect the average slope determines the maximum error, since we assume that the initial strain is unknown. Equation (4) gives the value of the deviation, and its maximum value corresponds to $\delta \varepsilon$ $=T_{\varepsilon} / 2$. For example, in the case of $d=1 \mathrm{~mm}\left(T_{\varepsilon}=\right.$ $355 \mu \varepsilon, \lambda_{p} \approx 1.545 \mu \mathrm{m}, B=0.753$ and $\left.d_{0}=1.2 \mathrm{~mm}\right)$ we find a maximum deviation of $\pm B \quad \lambda_{p}\left(T_{\varepsilon} / 2\right)$ $d /\left(d+d_{0}\right)= \pm 90 \mathrm{pm}$, and according to the chirp of the grating, the error in the position $z$ would be $\pm 3 \mathrm{~mm}$. However, once a first approximation is obtained, adjusting the numerical simulations to match the experimental dependence with the applied strain, it should be possible to refine the location of the crack. We can also analyse how precisely we can obtain the information on crack position from the measurement of the group delay when applying an external strain. Fig. 13 summarizes the results that we have obtained. As an example we have chosen the case $d=1 \mathrm{~mm}$. First, in Fig. 13 (a) we can see that $\tau_{g}$ follows a sort of horizontal saw-tooth curve, which average is about the $\tau_{g}$ value that we would get from the basic analysis carried out in section 3.2 (see Fig. 7 (b)). Second, in Fig. 13 (b) we can see that $\tau_{g p}$ has a periodic behavior with a period that matches the period of the staircase $\left(T_{\varepsilon}\right)$ defined in Section $4, T_{\varepsilon}=355 \mu \varepsilon$ for $d=1 \mathrm{~mm}$. So now again, we can correlate the dependence of the group delay versus the applied strain with the FabryPerot effects commented in the previous section.

From the point of view of determining the crack position in a realistic experiment, and assuming that the curing process of the material generates an unknown strain, the extreme peak values of the sawtooth response depicted in Fig. 13 (a) determines the error. In this example, the values of $\tau_{g}$ are within a deviation of \pm 20 ps with respect the average, and according to the group delay of the CFBG (10 $\mathrm{ps} / \mathrm{mm})$, this would correspond to an uncertainty in the determination of the spatial position of the crack of $\pm 2 \mathrm{~mm}$. This value is in good agreement with the value obtained when discussing the determination of the crack position after the spectral position of the resonance. Again, if the measurement of $\tau_{g}$ versus applied strain is feasible, then with this extra information one could adjust the simulations with the experiment and obtain a more accurate determination of the spatial location of the crack.

\section{Preliminary experimental verification}

In Fig. 14, we present a scheme of the experimental setup employed for a preliminary verification of the use of a CFBG for the detection of cracks. In our approach, there was no embedded CFBG, but an alternative experimental arrangement in which a short section of the CFBG is covered with a drop of glue, and this gives rise to a differential strain when a stress is applied to the fiber.

The CFBG was recorded in photosensitive SM fiber using a doubled Ar laser (244 nm) and a $50 \mathrm{~mm}$ uniform phase mask. The moving fiber - scanning beam approach was used to generate the chirp [16]. A segment of photosensitive optical fibre containing a $50 \mathrm{~mm}$ long CFBG was fixed at two points (A and $\mathrm{B})$, one of which (B), was attached to a micrometric translation stage in order to apply a known strain.

The CFBG had a spectral width of $1.105 \mathrm{~nm}$ (corresponding to a chirp $C=22.1 \mathrm{pm} / \mathrm{mm}$, and a chromatic dispersion of $403 \mathrm{ps} / \mathrm{nm}$ ), the central wavelength was $1543.78 \mathrm{~nm}$. The defect was located approximately in the centre of the CFBG at $z=25$ $\mathrm{mm}$.

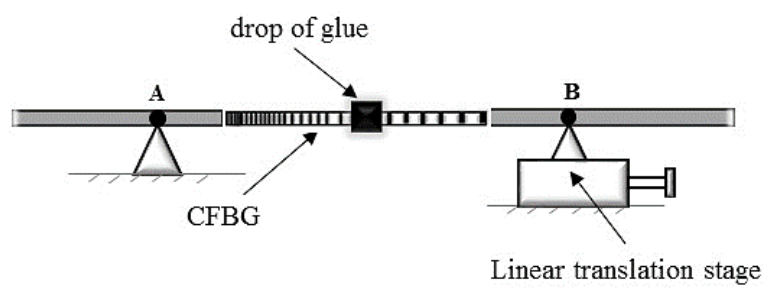

Fig. 14. Scheme of the experimental setup. The drop of glue generates the differential strain, simulating the presence of a crack in an embedded CFBG. 
Fig. 15 (a) shows the experimental spectrum of reflectivity and group delay of the CFBG before stretching the fiber with the micrometer.

To measure the group delay of the CFBG, we employed the so-called phase shift technique [17], using a tunable laser (Photonetics TUNICS-BT) in conjunction with a vector voltmeter (Agilent 8508A), a signal generator (Agilent 8648A) and an electrooptic modulator.
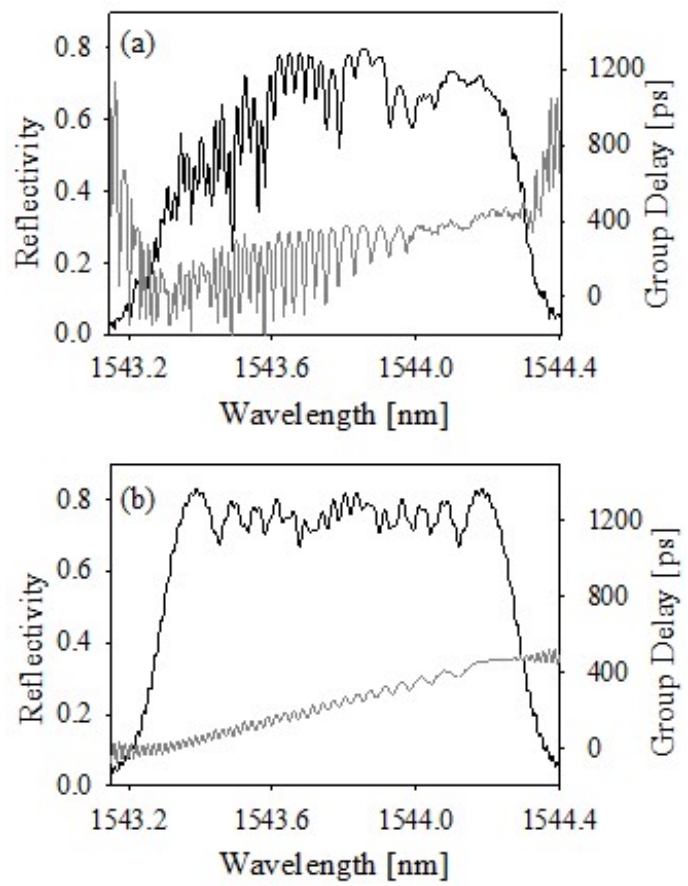

Fig. 15. Reflectivity (black line) and group delay (gray line) of CFBG at its initial condition $(\varepsilon=0)$ : (a) experiment, (b) simulation.

The grating shows a maximum reflectivity of $80 \%$. Fig. 15 (b) gives the theoretical values that have been computed adjusting the refractive index modulation in order to match the experimental reflectivity ( $\delta n=$ $9.3 \times 10^{-5}, \Lambda_{i}=514.42 \mathrm{~nm}, \Lambda_{f}=514.77 \mathrm{~nm}, n_{\text {eff }}=1.5 \mathrm{y}$ $L=5 \mathrm{~mm}$ ). We can see a good agreement with the experiment, although the experimental CFBG appears to have a reflectivity somewhat smaller at the blue part of the spectrum.

Figure 16 shows the spectra of the CFBG when a strain of $523 \mu \varepsilon$ was applied. It is clearly observed the generation of the resonances in both the amplitude and the group delay spectra. The theoretical simulation was computed with the same parameters than in Fig. 15 (b). A good match between theory and experiment can be observed.
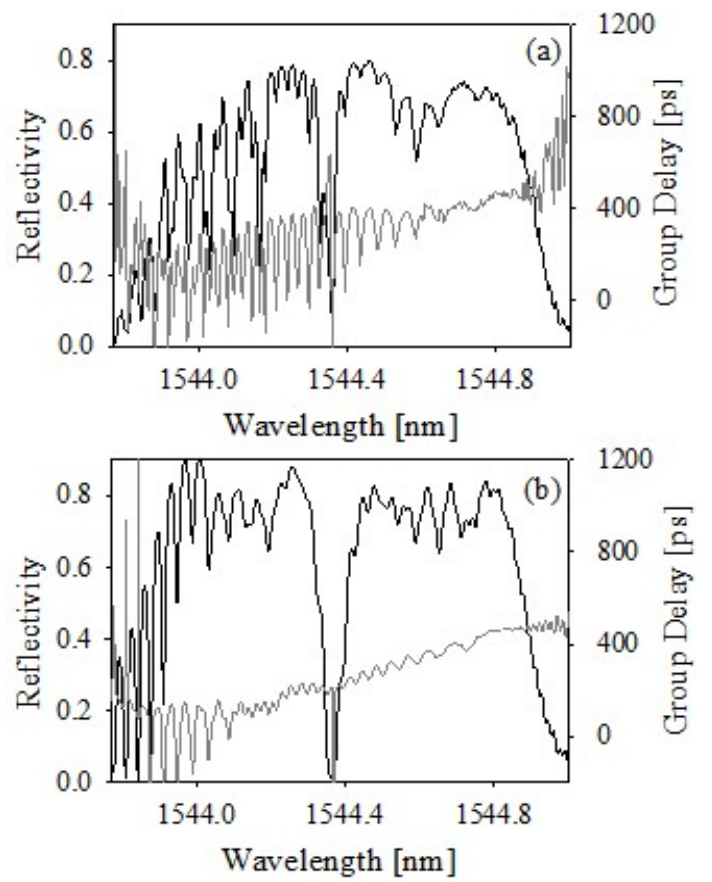

Fig. 16. Reflectivity (black line) and group delay (gray line) at $\varepsilon=523 \mu \varepsilon$ : (a) experiment, (b) simulation.

Fig. 17 represents the spatial position $(z)$ versus the wavelength difference, i. e., the nominal chirp of the grating as it was fabricated, and the 2 experimental values obtained after the measurement of the amplitude and group delay spectra for the strain values 523 and $1300 \mu \varepsilon$. In this figure the wavelength difference $\Delta \lambda^{\prime}$ is defined from the right border of the CFBG $\left(\lambda_{d \varepsilon}\right)$ to the wavelength position of the transmission peak $\left(\lambda_{p}\right): \Delta \lambda^{\prime}=\lambda_{d \varepsilon}-\lambda_{p}$. We observe that the experimental values of $\Delta \lambda^{\prime}$ for two strain values remain approximately constant, and according to the slope determined by the chirp, the spatial position of the crack would be $23 \mathrm{~mm}$, matching correctly the actual value fixed by the experimental arrangement. 


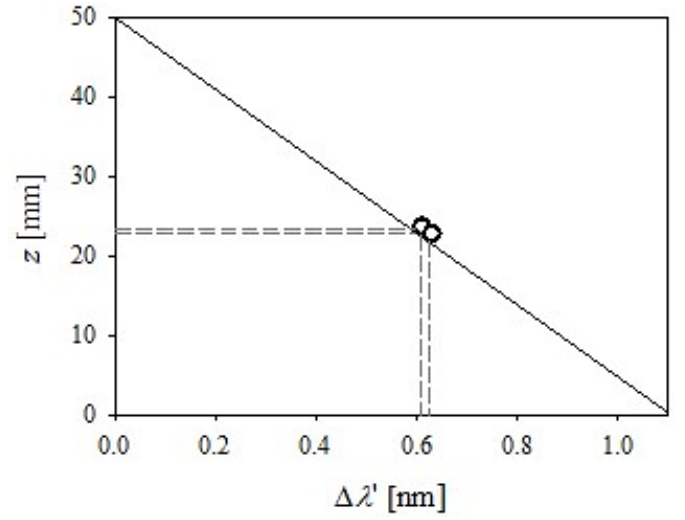

Fig. 17. Chirp of the grating as it is defined in the fabrication process (solid line) and experimental points determined by the wavelength difference of the transmission peak with respect to the long wavelength border of the grating $\left(\Delta \lambda^{\prime}\right)$.

Although we only have experimental measurements for 2 values of strain (523 and $1300 \mu \varepsilon$ ) we have proceeded to compare the two experimental points with the simulated dependence of the resonance wavelength with applied strain, by plotting the strain versus wavelength. We can see that the two experimental points match perfectly the computed response by adjusting the width $(d=1.6 \mathrm{~mm})$. Although the nominal value of $d$ was $2 \mathrm{~mm}$, we find a better agreement with a slightly smaller value. With this adjusted value, we observe as well a good match with the actual amplitude of the resonance which is high at $523 \mu \varepsilon$, but rather small at $1300 \mu \varepsilon$ (Fig. 18).

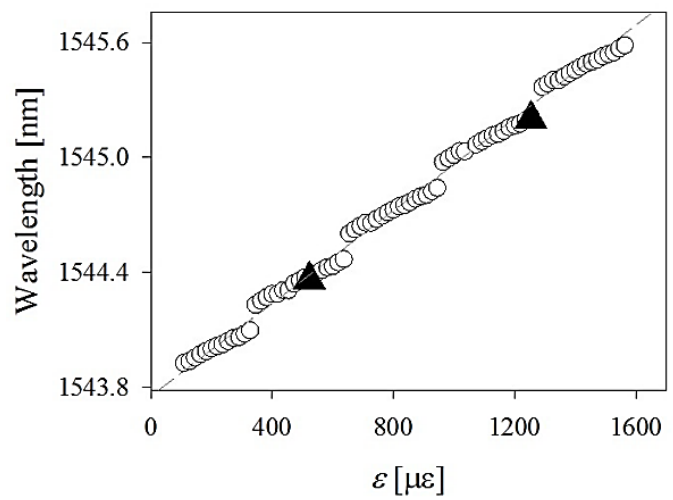

Fig. 18. Wavelength of the transmission peak versus strain. The two experimental points are the triangles. Simulations performed for a defect $\mathrm{d}=1.6 \mathrm{~mm}$.

\section{Conclusions}

The results of the present work demonstrate that the use of CFBG for the characterization of cracks is not straightforward and it is not possible to perform the analysis with a simple correlation between spectral position of resonances and nominal chirp of the grating. First, we find that a single crack can produce several peaks in the grating spectral response and, second, we find that as a function of the remaining strain the spectral position of the peak changes significantly. Our results show that by measuring the response versus applied strain one can overtake the main limitations, since the slope of the peaks' shift versus strain gives direct information on the crack width and enables a precise determination of the crack position. In addition, the measurement of the group delay versus strain gives redundant information that can be used for verification purposes or as an alternative to the measurement of transmission or reflection spectra. According to our knowledge, previously published works do not refer to the possibility of measuring crack width, so this is an original contribution of the research work presented here. Although some experimental results are provided, a precise experimental test is still required in order to confirm the model with detail.

\section{Acknowledgements}

This work has been supported by CONICET (PIP 112-201101-00397), Facultad de Ingeniería Universidad Nacional de La Plata (Project I169/12) and Comisión de Investigaciones Científicas de la Provincia de Buenos Aires - CIC (Resolución No 1266/15), Argentina. A. Mesa Yandy is CIC fellow, R. Duchowicz and N. A. Russo are researchers of CONICET and CIC, respectively.

J. L. Cruz and M. V. Andrés acknowledge financial support from the Ministerio de Economía y Competitividad of Spain and FEDER funds (Ref.: TEC2016-76664-C2-1-R) and the Generalitat Valenciana of Spain (Ref.: PROMETEOII/2014/072). 


\section{References}

[1] P. Bettini, E. Guerreschi, and G. Sala, "Development and experimental validation of a numerical tool for structura health and usage monitoring systems based on chirped grating sensors," Sensors (Switzerland), vol. 15, no. 1, pp. 1321-1341, 2015.

[2] X. W. Ye, Y. H. Su, and J. P. Han, "Structural Health Monitoring of Civil Infrastructure Using Optical Fiber Sensing Technology: A Comprehensive Review.," Sci. World J., vol. 2014, p. 652329, 2014.

[3] D. Kinet, P. Mégret, K. W. Goossen, L. Qiu, D. Heider, and C. Caucheteur, "Fiber Bragg grating sensors toward structural health monitoring in composite materials: challenges and solutions," Sensors, vol. 14, no. 4, pp. 7394-7419, 2014.

[4] Y. Okabe, R. Tsuji, and N. Takeda, "Application of chirped fiber Bragg grating sensors for identification of crack locations in composites," Compos. Part A Appl. Sci. Manuf., vol. 35, no. 1, pp. 59-65, 2004.

[5] J. Palaniappan et al., "Prediction of the reflected spectra from chirped fibre Bragg gratings embedded within cracked crossply laminates," Meas. Sci. Technol., vol. 17, no. 6, pp. 1609-1614, 2006.

[6] J. Palaniappan et al., "Changes in the reflected spectra of embedded chirped fibre Bragg gratings used to monitor disbonding in bonded composite joints," Compos. Sci. Technol., vol. 67, no. 13, pp. 2847-2853, Oct. 2007.

[7] R. L. Rito, A. D. Crocombe, and S. L. Ogin, "Health monitoring of composite patch repairs using CFBG sensors: Experimental study and numerical modelling," Compos. Part A Appl. Sci. Manuf., vol. 100, pp. 255268, Sep. 2017.

[8] C. A. F. Marques, P. Antunes, P. Mergo, D. J. Webb, and P. Andre, "Chirped Bragg Gratings in PMMA StepIndex Polymer Optical Fiber," IEEE Photonics Technol. Lett., vol. 29, no. 6, pp. 500-503, 2017.

[9] C. A. F. Marques et al., "Chirped polymer optical fiber Bragg grating sensors," Proc. SPIE - Int. Soc. Opt. Eng., vol. 10232, no. May 2017, 2017.

[10] R. Kashyap, Fiber Bragg gratings. San Diego, California, 2010

[11] R. Kashyap and M. de Lacerda Rocha, "On the group delay characteristics of chirped fibre Bragg gratings," Opt. Commun., vol. 153, no. 1-3, pp. 19-22, Jul. 1998.

[12] Y. O. Barmenkov, D. Zalvidea, S. Torres-Peiró, J. L. Cruz, and M. V. Andrés, "Effective length of short Fabry-Perot cavity formed by uniform fiber Bragg gratings," Opt. Express, vol. 14, no. 14, p. 6394, 2006.

[13] X. Roselló-Mechó, M. Delgado-Pinar, A. Díez, and M. V. Andrés, "Measurement of Pockels' coefficients and demonstration of the anisotropy of the elasto-optic effect in optical fibers under axial strain," Opt. Lett., vol. 41, no. 13 , p. 2934, 2016.

[14] A. D. Kersey et al., "Fiber grating sensors," J. Light. Technol., vol. 15, no. 8, pp. 1442-1463, 1997

[15] Y. O. Barmenkov, A. V. Kir'yanov, P. Pérez-Millán, J. L. Cruz, and M. V. Andrés, "Experimental study of a symmetrically-pumped distributed feed-back Erbiumdoped fiber laser with a tunable phase shift," Laser Phys. Lett., vol. 5, no. 5, pp. 357-360, 2008
[16] W. H. Loh, M. J. Cole, M. N. Zervas, S. Barcelos, and R. I. Laming, "Complex grating structures with uniform phase masks based on the moving fiber-scanning beam technique.," Opt. Lett., vol. 20, no. 20, pp. 2051-2053, 1995.

[17] G. Cancellieri, "Single-mode optical fiber measurement: Characterization and Sensing," Artech House, Inc., pp. 141-142, 1993. 